\title{
Age-dependent alpha-synuclein accumulation and aggregation in the colon of a transgenic mouse model of Parkinson's disease
}

\author{
Qian-Qian Chen ${ }^{1}$, Caroline Haikal ${ }^{2}$, Wen Li ${ }^{2}$, Ming-Tao Li ${ }^{3}$, Zhan-You Wang ${ }^{4}$ and Jia-Yi Li ${ }^{1,2^{*}}$
}

\begin{abstract}
Background: Parkinson's disease (PD) is one of the most common neurodegenerative diseases, neuropathologically characterized by misfolded protein aggregation, called Lewy bodies and Lewy neurites. PD is a slow-progressive disease with colonic dysfunction appearing in the prodromal stage and lasting throughout the course of the disease.

Methods: In order to study PD pathology in the colon, we examined the age-dependent morphological and pathological changes in the colon of a PD mouse model expressing human wildtype a-synuclein (a-syn) fused with the green fluorescent protein (GFP), under the endogenous mouse a-syn promoter.

Results: We observed an age-dependent progressive expression and accumulation of a-syn-GFP in the enteric neurons of Meissner's (submucosal) and Auerbach's (myenteric) plexuses of the colon. Additionally, the phosphorylation of a-syn at serine 129 also increased with age and the aggregation of a-syn-GFP coincided with the appearance of motor deficits at 9 months of age. Furthermore, a-syn (-GFP) distinctly co-localized with different subtypes of neurons, as identified by immunohistochemical labeling of vasoactive intestinal peptide (VIP), neuronal nitric oxide synthase (nNOS), and calretinin.

Conclusions: Our results show the development of a-syn pathology in the enteric neurons of the colon in a PD mouse model, which coincide with the appearance of motor deficits. Our mouse model possesses the potential and uniqueness for studying PD gastrointestinal dysfunction.
\end{abstract}

Keywords: Parkinson's disease, Colon, a-syn, Phosphorylation, VIP, nNOS, Calretinin, Enteric nervous system

\section{Background}

Parkinson's disease (PD) is a common neurodegenerative disease of ageing, pathologically characterized by the loss of dopaminergic neurons in the substantia nigra pars compacta [1] and the formation of intracellular inclusions termed Lewy bodies (LBs) and Lewy neurites (LNs) $[2,3]$. Immunohistochemical analyses have shown that misfolded $\alpha$-syn is the primary protein component

\footnotetext{
* Correspondence: jiayili@mail.neu.edu.cn; jia-yi.li@med.lu.se

${ }^{1}$ Institute of Neuroscience, College of Life and Health Sciences, Northeastern University, 110819 Shenyang, People's Republic of China

${ }^{2}$ Neural Plasticity and Repair Unit, Wallenberg Neuroscience Center,

Department of Experimental Medical Science, Lund University, BMC A10,

22184 Lund, Sweden

Full list of author information is available at the end of the article
}

of LBs and LNs [4], which suggests its potential role in the pathogenesis of PD. Typical motor symptoms of PD include tremor, rigidity, bradykinesia, and postural instability. Most, if not all, PD patients also suffer from multiple non-motor features such as depression, rapid eye movement sleep behavioral disorder, excessive daytime sleepiness, constipation, etc [5]

Recent evidence suggests that PD pathology can spread from the gut to the brain [6]. Braak and colleagues first proposed this hypothesis based on their systemic examinations of tissues from the peripheral organs to the brain: Lewy pathology in the intestinal tract and lower brain stem preceded the appearance of pathology in the upper brain regions in postmortem tissues of PD patients $[7,8]$.

(C) The Author(s). 2018 Open Access This article is distributed under the terms of the Creative Commons Attribution 4.0 International License (http://creativecommons.org/licenses/by/4.0/), which permits unrestricted use, distribution, and 
Recently, we showed direct evidence of PD pathology spread from the gastrointestinal tract to the brain in a rat model after exogenous $\alpha$-syn was injected into the intestinal wall [6]. In addition, retrospective statistical analyses showed that full truncal vagotomy diminished the risk of subsequent PD development, suggesting that the vagal nerve, the neuronal connection between the gut and brain, is a route for PD pathology spread [9]. Moreover, our previous study demonstrated that $\alpha$-syn was more highly expressed in the distal segment of the small intestine than in the proximal segment. It is consistent with the distribution of the microbiota that: the stomach and upper small intestine are essentially sterile; the bacteria count rises further along the intestine [10]. Considering that the large intestine contains the largest bacterial ecosystem in the human body [11], it inspired us to further explore whether pathological alteration of $\alpha$-syn is present in the enteric nervous system of the colon, which plays an essential role in the development of constipation in early PD.

\section{Materials and Methods Mouse strain}

The transgenic mouse model used in this study has been described previously $[12,13]$. Briefly, BAC- $\alpha-$ syn-GFPtransgenic mice were generated by pronuclear injection of BAC DNA into fertilized eggs of C57 black 6 (C57BL/ 6) mice. The BAC DNA is composed of the human full-length wildtype $\alpha$-syn gene fused with the GFP sequence. The human $\alpha$-syn cDNA was inserted into the pAcGFP1-C1 vector at the initiation codon of the mouse $\alpha$-syn gene.

\section{Tissue preparation}

BAC mice aged 3 to 24 months $(3 \mathrm{~m}, n=3 ; 6 \mathrm{~m}, \mathrm{n}=3$; $9 \mathrm{~m}, \mathrm{n}=3 ; 18 \mathrm{~m}, n=1 ; 24 \mathrm{~m}, \mathrm{n}=3$ ) and C57BL/6 wildtype mice $(6 \mathrm{~m}, \mathrm{n}=3 ; 9 \mathrm{~m}, \mathrm{n}=3 ; 18 \mathrm{~m}, \mathrm{n}=3)$ were sacrificed by transcardial perfusion with $0.1 \mathrm{M}$ phosphate buffer solution (PBS), followed by $4 \%$ paraformaldehyde (PFA). The colon was dissected and post-fixed in 4\% PFA overnight. Fixed colon was then transferred to 10, 20,30\% sucrose in $0.1 \mathrm{M}$ PBS, consecutively. The colon was then cut transversely into $10 \mu \mathrm{m}$ sections using a cryostat (Leica, Germany) and mounted on gelatin-coated glass slides.

\section{Immunohistochemistry and analyses}

Antigen retrieval of the samples was performed in heated citrate buffer (pH 6). Endogenous peroxidase was blocked by treatment with $3 \%$ hydrogen peroxide and $10 \%$ methanol in $0.1 \mathrm{M}$ PBS at room temperature for $15 \mathrm{~min}$. The sections were then pre-incubated with blocking solution (5\% normal goat serum and $0.3 \%$ Triton X 100 in $0.1 \mathrm{M}$ PBS) for $1 \mathrm{~h}$ at room temperature. Primary antibody (anti- $\alpha$-syn (phosphoS129), Abcam, ab51253, rabbit, dilution: $1 / 500$ ), was incubated on samples at $4{ }^{\circ} \mathrm{C}$ overnight, followed by incubation with the secondary antibody (biotinylated goat anti-rabbit IgG antibody, Vector Laboratories, BA-1000, dilution: 1/500) at room temperature for $1 \mathrm{~h}$, and $\mathrm{ABC}$ solution (Vector Bio labs, Cat. \# VEPK-6100) at room temperature for $1 \mathrm{~h}$. The staining was developed with diaminobenzidine (VMR chemical, Cat. \# 0430, 0.03\% dissolved in $0.05 \mathrm{M} \mathrm{pH} 7.5$ tris- $\mathrm{HCl}$ ) for $5 \mathrm{~min}$. The sections were then dehydrated sequentially with $70,80,90$, $95,100 \%$ ethanol and dimethyl benzene, then mounted with neutral balsam for microscopic analyses. In each round of immunohistochemical staining, we included "negative controls" without incubation of the primary antibodies (Additional file 1: Figure S1C).

For double fluorescent immunostaining, the process of the antigen retrieval completely quenched the transgenically expressed GFP (Additional file 1: Figure S1A). After pre-incubation with blocking solution (5\% normal donkey serum and $0.3 \%$ Triton X 100 in $0.1 \mathrm{M} \mathrm{PBS}$ ), the sections were incubated with primary antibodies (i.e. $\alpha$-syn antibody with the respective specific neuronal marker antibodies) and their paired double staining antibodies (Table 1) at room temperature for $1 \mathrm{~h}$. The sections were mounted with an anti-fading medium for confocal microscopic analysis (Leica TCS SP8).

Quantification of the intensity of $\alpha$-syn-GFP, calretinin, VIP and nNOS immunofluorescence was performed by measuring the mean fluorescence intensity in the positive area of the colonic cross-sections. The measurement of phospho- $\alpha$-syn intensity was based on the gray value of immunohistochemistry staining in Auerbach's plexus. The measurements of co-localization were processed using the Pearson's Correction with the Fiji plugin Coloc 2. Two-way ANOVA tests were performed to statistically analyze the immunofluorescence intensity and co-localization, followed by Bonferroni adjustment post hoc tests with the software IBM SPSS Statistics 2.0.

\section{Results \\ Age-dependent expression and accumulation of a-syn- GFP in the enteric neurons of the colon}

We first analyzed the expression of $\alpha$-syn-GFP in the colon in the proximal (close to the cecum) and distal (close to the rectum) regions. $\alpha$-Syn-GFP was present in the nerve terminals in the mucosal layer and Auerbach's and Meissner's plexuses (Additional file 1: Figure S1B). The expression of $\alpha$-syn-GFP was age-dependent; the transgene expression of $\alpha$-syn-GFP in both the proximal and distal colon increased with age (Fig. 1). At 3 months of age, $\alpha$-syn-GFP was weakly and sparsely present in the nerve fibers of the myenteric and the mucosal layers. From 6 months onwards, $\alpha$-syn-GFP signals became more evident in the nerve fibers both in the myenteric and mucosal layers, and around the crypts of Lieberkühn (Fig. 1). 
Table 1 Primary and secondary antibodies in double immunostainings

\begin{tabular}{lll}
\hline Double Immunostaining & 1st antibodies & 2nd antibodies \\
\hline a- syn \& PGP .5 & Anti-a-syn: Santa Cruz Biotechnology, sc-12,767, & Cy3-donkey anti-mouse: \\
& mouse, dilution: 1/200 & Jackson ImmunoResearch, 715-165-150, dilution:1/200 \\
& Anti-PGP 9.5: Abcam, ab72910, & Alexa 488 goat anti-chicken: \\
& Chicken, dilution:1/800 & Invitrogen, A11039, dilution: 1/1000 \\
a- syn \& a-syn (phosphoS129) & Anti-a-syn: Santa Cruz Biotechnology, sc-12,767, & Alexa 488-donkey anti-mouse: \\
& mouse, dilution: 1/200 & Jackson ImmunoResearch, 715-546-151, dilution:1/200 \\
& Anti-a-syn (phosphoS129): Abcam, ab51253, & Cy3-donkey anti-rabbit: Jackson ImmunoResearch, \\
& rabbit, dilution:1/200 & 711-165-152, dilution:1/800 \\
& Anti-a-syn: Santa Cruz Biotechnology, sc-7011-r, & Cy3-donkey anti-rabbit: \\
& rabbit, dilution: 1/200 & Jackson ImmunoResearch, 711-165-152, dilution:1/800; \\
& Anti-calretinin: Santa Cruz Biotechnology, sc-11,644, & Alexa 488-donkey anti-goat: \\
& goat, dilution:1/400 & Jackson ImmunoResearch, 705-545-147, dilution:1/400 \\
& Anti-a-syn: Santa Cruz Biotechnology, sc-12,767, & Alexa 488-donkey anti-mouse: \\
& mouse, dilution: 1/200 calretinin & Jackson ImmunoResearch, 715-546-151, dilution:1/200 \\
a- syn \& nNOS & Anti-neuronal nitric oxide synthase: Abcam, ab76067, & Cy3-donkey anti-rabbit: \\
& rabbit, dilution:1/400 & Jackson ImmunoResearch, 711-165-152, dilution:1/800 \\
& $\begin{array}{l}\text { Anti-a-syn: Santa Cruz Biotechnology, sc-12,767, } \\
\text { mouse, dilution: 1/200 }\end{array}$ & Alexa 488-donkey anti-mouse: \\
Anti-vasoactive intestinal polypeptide: Abcam, ab22736, & Jackson ImmunoResearch, 715-546-151, dilution:1/200 \\
a- syn \& VIP & rabbit, dilution:1/800 & Jacksonkey ImmunoResearch, 711-165-152, dilution:1/800 \\
\hline
\end{tabular}

\section{Age-dependent phosphorylation of a-syn in the enteric neurons of the colon}

As one of the most common post-translational modifications, phosphorylation of $\alpha$-syn plays an important role in PD pathogenesis. We examined the status of $\alpha$-syn phosphorylation (ser 129) in the enteric neurons of the colon of BAC- $\alpha$-syn-GFP mice at different ages. At 6 months of age, phosphorylated $\alpha$-syn was rarely detected in the enteric neurons in the proximal and distal colons of the mice (Fig. 2a). However, with age, phospho- $\alpha$-syn became more abundant (Fig. 2d). $\alpha$-Syn phosphorylation was more robust in the nerve fibers and terminals in Auerbach's (myenteric) plexus, compared to Meissner's plexus. Interestingly, phospho- $\alpha$-syn containing cell bodies of myenteric neurons were observed
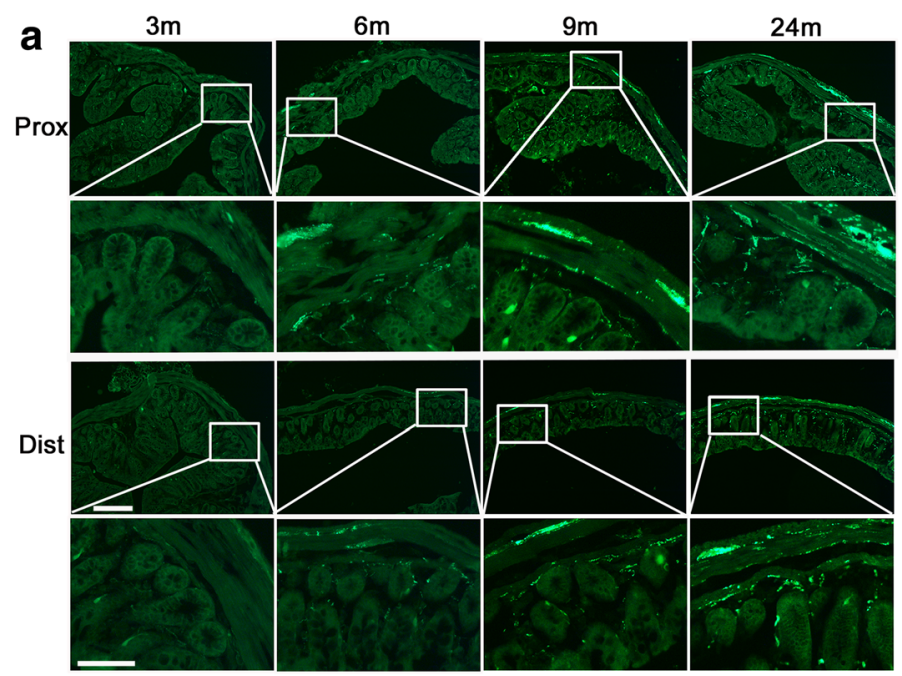

b
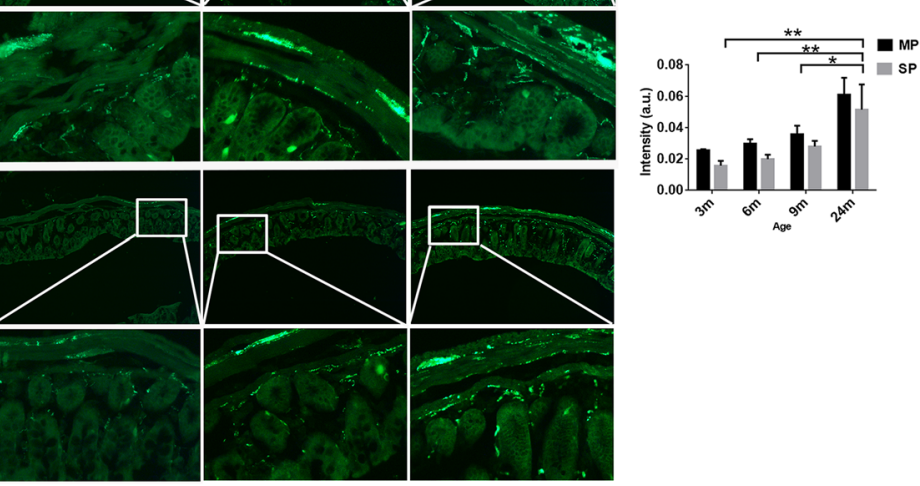

Fig. 1 The presence of a-syn in enteric neurons of the colon with age. a. Representative images of a-syn-GFP transgene expression in the proximal (Upper panels) and distal (Lower panels) colon at 3, 6, 9 and 24 months of mice. $\mathbf{b}$. The semi-quantitative analyses of a-syn fluorescence (GFP) intensity in the colon of transgenic mice at different ages [two-way ANOVA ( $F=9.233, p=0.001$, in ages. $F=0.089, p=0.98$ ), followed by Bonferroni adjustment post hoc tests $\left.\left({ }^{* * *} p<0.001,{ }^{* *} p<0.01,{ }^{*} p<0.05\right)\right]$. Error bars in SEM. Scale bar $=250 \mu \mathrm{m}$ and $100 \mu \mathrm{m}$ (zoomed up images) in a 


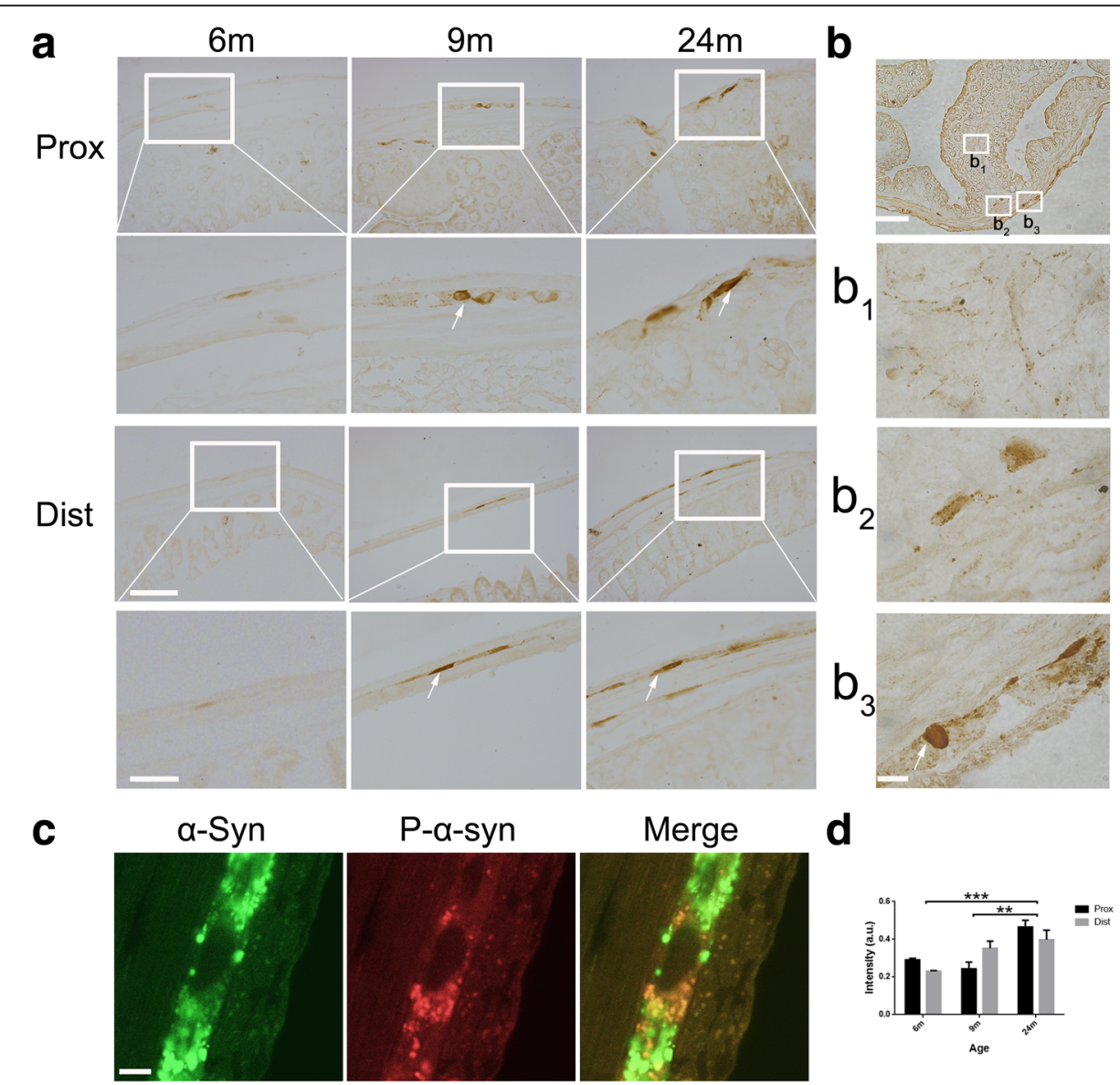

Fig. 2 The accumulation of phospho-a-syn (pS129) in enteric neurons of the colon. a. The age-dependent phosphorylation of a-syn in the Auerbach's plexus of the proximal (Upper panels) and distal (Lower panels) colon. b. Phospho-a-syn in the colon at the age of 18 months ( $b_{1}$, Mucosal layer. $b_{2}$, Meissner's plexus. $b_{3}$, Auerbach's plexus). c. Partial phosphorylation (phospho-a-syn (red)) in the Auerbach's plexus, viewed as pan a-syn expression (green) (Pearson's correction:0.388 \pm 0.100). $\mathbf{d}$. The semi-quantitative analyses of phospho-a-syn intensity (immunohistochemical staining) in the Auerbach's plexus. Two-way ANOVA ( $F=14.69, p=0.0006$, in ages. $F=0.5055, p=0.8259)$, followed by Bonferroni adjustment post hoc tests ( ${ }^{* * *} p<0.01$, ${ }^{* *} p<0.001$ ). Arrows show phospho-a-syn containing cell bodies of myenteric neurons. Scale bars $=250 \mu \mathrm{m}$ and $100 \mu \mathrm{m}$ (zoomed up images) in a; $500 \mu \mathrm{m}$ and $50 \mu \mathrm{m}$ in $\mathbf{b}$; and $10 \mu \mathrm{m}$ in $\mathbf{c}$

(Fig. 2a and b, arrows). Partial co-localization of pan $\alpha$-syn and phospho- $\alpha$-syn was detected in Auerbach's plexus (Fig. 2c).

\section{a-Syn(-GFP) in subsets of enteric neurons in the colon}

Different subtypes of neurons secrete different transmitters and regulate colonic function. Calretinin plays an important role in PD: striatal calretinin neurons are sensitive to dopamine depletion and calretinin in dopaminergic neurons may have a protective function for their survival [14-16]. The number of nNOS-expressing interneurons is up-regulated in PD and nNOS is thus implicated in the disease pathogenesis [17]. On the other hand, the treatment with VIP can reverse the motor deficits in the 6-hydroxydopamine (6-OHDA) lesioned model of PD [18]. Thus, we analyzed these three subtypes of neurons in the colon by double immunofluorescence labeling with the $\alpha$-syn antibody and the respective neuronal subtype-specific marker. We observed that calretinin was widely expressed in both Auerbach's and Meissner's plexuses and nerve fibers in different colonic layers. The expression of calretinin increased with age (Fig. 3a, b and d). Calretinin and $\alpha$-syn (-GFP) highly co-localized in the two plexuses and in the nerve fibers of all the layers (mucosal, submucosal and myenteric layers) (Fig. 3c and f). the extent of co-localization increased with age (Fig. 3e). Similarly, VIP was broadly expressed in both Auerbach's and Meissner's plexuses and nerve fibers in different colonic layers and it was increased in both the proximal and distal colon with age (Fig. 4a, b and d). VIP was highly co-localized with $\alpha$-syn (-GFP) in the mucosal layer fibers and Meissner's plexus, but, to a lesser extent, in Auerbach's plexus (Fig. 4c and f). nNOS, on the other hand, was expressed in Auerbach's and Meissner's plexuses and in nerve fibers of the muscular layer (Fig. 5a and b), but not present in the nerve fibers in submucosal and mucosal layers. The expression of nNOS in the 


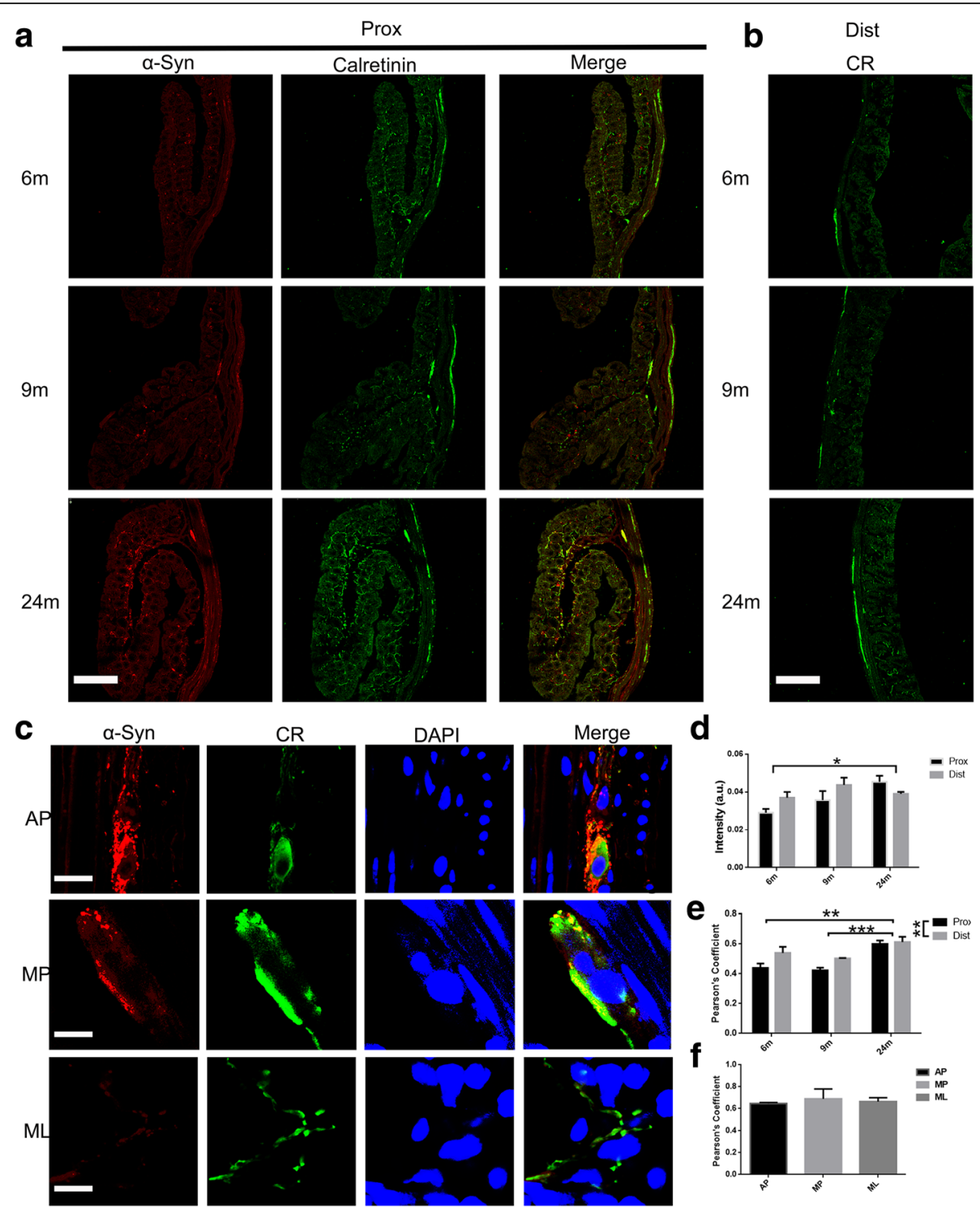

Fig. 3 Co-localization between calretinin and a-syn in enteric neurons in the colon. a a-syn and calretinin expression in the enteric neurons increased with age and highly co-localized to each other at 6, 9, and 24 months in the proximal colon. $\mathbf{b}$ Expression of calretinin in the distal colon also increased with age. c Co-localization between calretinin (CR) and a-syn in the enteric nerve terminals and neuronal cell bodies in the mucosal layers (ML) and Auerbach's (AP) and Meissner's plexuses (MP) at 24 months. $\mathbf{d}$ Semi-quantitative analyses of calretinin in the colon of BAC a-syn-GFP mice. Two-way ANOVA ( $F=6.277, p=0.014$, in ages. $F=0.420, p=0.529$ ), followed by Bonferroni adjustment post hoc tests ( $\left.{ }^{*} p<0.05\right)$. e Quantitative of co-localization between calretinin and $a$-syn in the colon in BAC $a$-syn-GFP mice. Two-way ANOVA ( $F=1.442, p=0.275$, in ages. $F=10.840, p=0.006)$. $\mathbf{f}$ Quantitative of co-localization between calretinin and a-syn in the mucosal layers (ML) and Auerbach's (AP) and Meissner's plexuses (MP) 24 months. Scale bar $=250 \mu m$ in $\mathbf{a}$ and $\mathbf{b} ; 20 \mu \mathrm{m}$ in $\mathbf{c}(\mathrm{MP}, \mathrm{ML})$ and $40 \mu \mathrm{m}$ in $\mathbf{c}(\mathrm{AP})$

proximal and distal colon showed no change with age (Fig. 5a, b and d), which is not consistent with the changes in the small intestine we previously reported [13]. Apart from this, $\mathrm{nNOS}$ was poorly co-localized with $\alpha$-syn (-GFP) in both Auerbach's and Meissner's plexuses (Fig. 5c and f).

\section{Discussion}

A large body of clinical reports have suggested that gastrointestinal dysfunction, such as constipation, is a common non-motor symptom in $\mathrm{PD}$, starting from the prodromal stage and lasting throughout the course of the disease $[19,20]$. The links between the gut and the brain are manifold and both direct and indirect in PD development. Braak and colleagues have suggested that $\alpha$-syn pathology could be initiated in the gut and then propagate to the brain. In support of this hypothesis, our lab has previously reported the direct spread of pathological $\alpha$-syn from the small intestine to the lower brain stem via the vagal nerve. Additionally, there have been studies suggesting a connection of PD progress and gut 


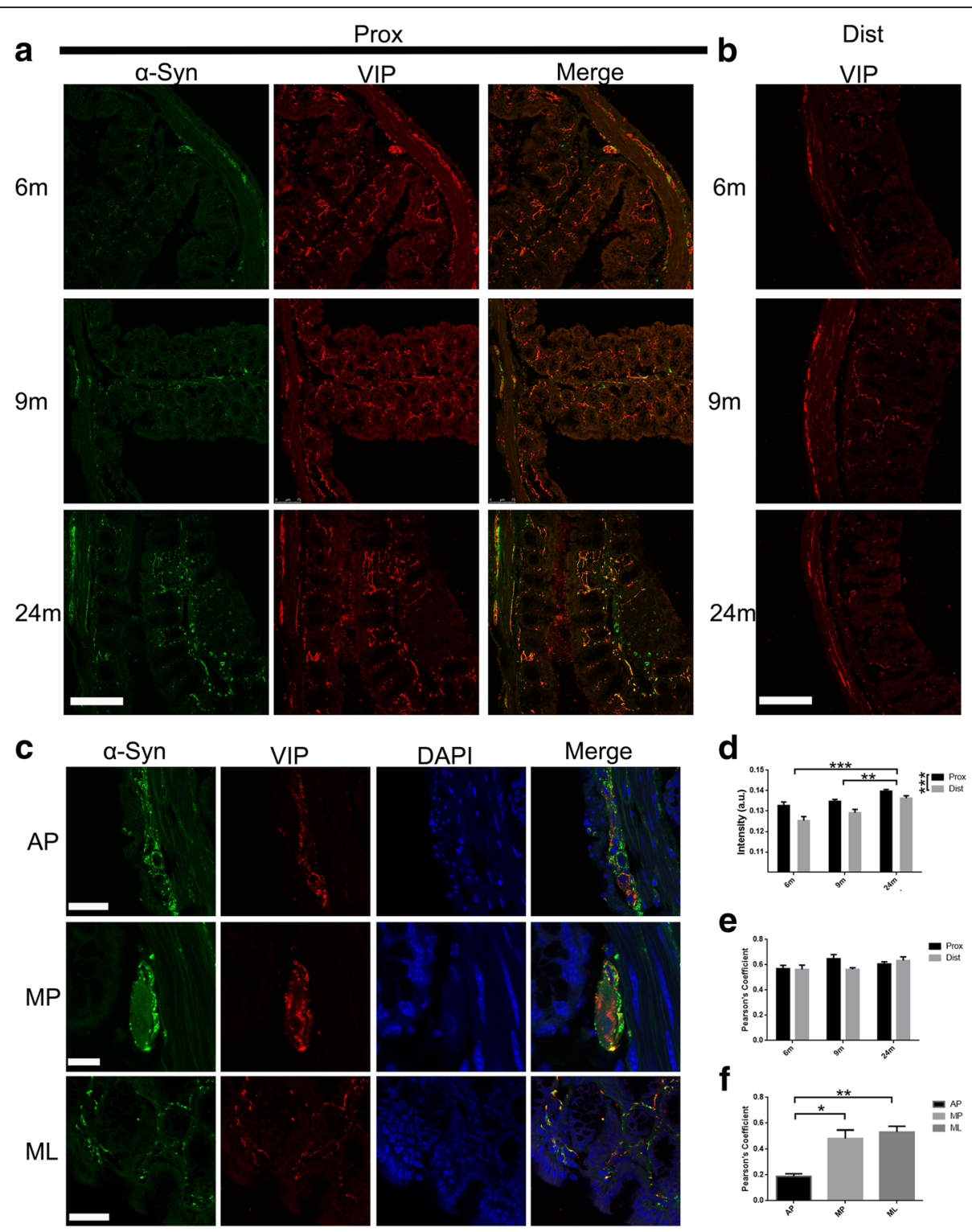

Fig. 4 Co-localization between VIP and a-syn in enteric neurons of the colon. $\mathbf{a}$ and $\mathbf{b}$, Age-dependent increase of VIP expression was observed in the enteric neurons of the proximal colon at 6, 9, and 24 months, partial co-localization of VIP and a-syn was seen at 24 months old of age. c Co-localization of VIP and a-syn, and the nucleus (DAPI) at 24 months in mucosal layers (MP) and Auerbach's (AP) and Meissner's (MP) plexuses. $\mathbf{d}$ Semi-quantitative analyses of VIP in the colon of BAC $a$-syn-GFP mice. Two-way ANOVA [F $=18.999, p=0.0002$, in ages. $F=19.950, p=0.0008\left({ }^{* * *} p\right)$, followed by Bonferroni adjustment post hoc tests $\left({ }^{* * *} p<0.001,{ }^{* *} P<0.01\right)$. e Quantitative of co-localization between VIP and a-syn in the colon in BAC a-syn-GFP mice. Two-way ANOVA ( $F=2.150, p=0.159$, in ages. $F=0.950, p=0.349)$ ]. $\mathbf{f}$ Quantitative of co-localization between VIP and $a-s y n$ at 24 months in mucosal layers (MP) and Auerbach's (AP) and Meissner's (MP) plexuses. Student $t$ test $\left({ }^{*} p<0.05,{ }^{* *} p<0.01\right)$. Scale bar $=150 \mu \mathrm{m}$ in a and $\mathbf{b} ; 50 \mu \mathrm{m}$ in $\mathbf{c}(\mathrm{AP}, \mathrm{ML})$ and $15 \mu \mathrm{m}$ in $\mathbf{c}(\mathrm{MP})$

inflammation. Bialecka et al. reported that the frequency of CARD15/NOD2 gene variants, associated with Crohn's disease, may be a risk factor for sporadic PD development [19]. In 2013, David et al. also found pro-inflammatory cytokines, glial fibrillary acidic protein and Sox-10 to be elevated in the ascending colon of PD patients [20]. Other studies have provided evidence of intestinal inflammation in PD patients through fecal examinations [21, 22]. Moreover, intestinal microbiota in PD patients were found to differ from those in healthy controls: a decrease of anti-inflammatory, butyrate-producing bacteria and an increase in pro-inflammatory bacteria were observed in PD patient stool samples compared to those of healthy controls [20, 21]. In experimental models, microbiota has similarly been shown to regulate 


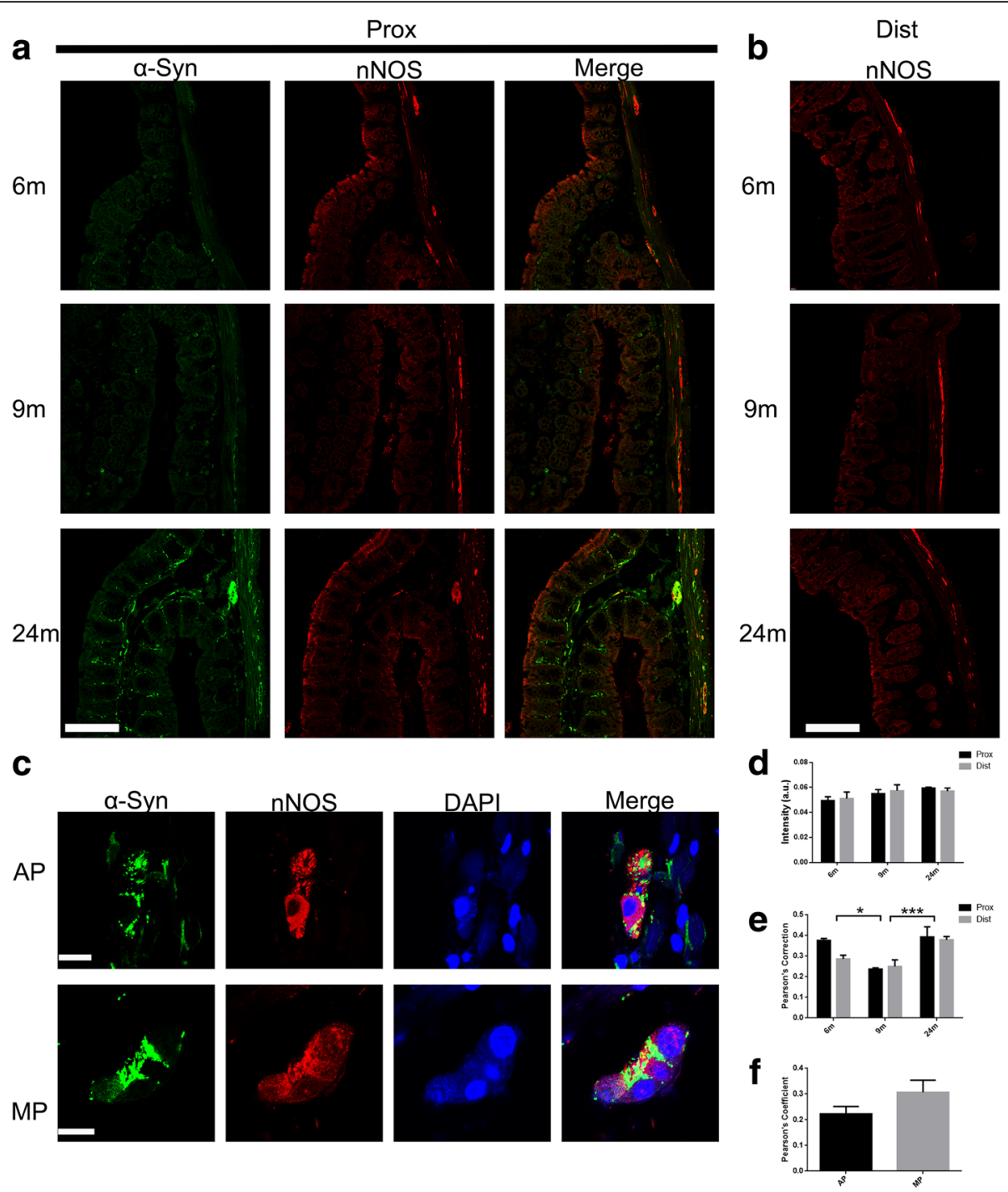

Fig. 5 Expression of nNOS in enteric neurons of the colon of transgenic mice and co-localization of nNOS and a-syn. a nNOS expression is predominant in the Auerbach's plexus without clear age-related changes. b Expression of nNOS in distal colon at 6, 9, and 24 months. c Colocalization of nNOS and a-syn, and the nucleus (DAPI) at 24 months in Auerbach's (AP) and Meissner's (MP) plexuses. $\mathbf{d}$ Semi-quantitative analyses of nNOS in the colon of BAC mice. Two-way ANOVA ( $F=2.723, p=0.106$, in ages. $F=0.031, p=0.862$ ). e Quantitative of co-localization between nNOS and $a-s y n$ in the colon in BAC mice. Two-way ANOVA ( $F=15.262, p=0.001$, in ages. $F=1.970, p=0.186)$, followed by Bonferroni adjustment post hoc tests $\left({ }^{*} p<0.05,{ }^{* * *} P<0.001\right)$. f Quantitative of co-localization between nNOS and a-syn at 24 months in Auerbach's (AP) and Meissner's (MP) plexuses. Scale bar $=150 \mu \mathrm{m}$ in $\mathbf{a}$ and $\mathbf{b}$; and $15 \mu \mathrm{m}$ in $\mathbf{c}$

movement deficits providing further support to the link between intestinal inflammation and PD pathology [22].

It is of importance to understand the involvement of the gastrointestinal system in initiation and development of PD pathology. Our BAC- $\alpha$-syn-GFP mice possess both the symptomatic and pathological features of early PD. $\alpha$-Syn-GFP accumulation and phosphorylation were found increased with age in the colon of the transgenic mice, which corresponds to our observations of an increased gut transit time (Additional file 2: Figure S2), implying a sign of constipation symptoms in mice. Regarding motor symptoms, in our previous study, the BAC- $\alpha$-syn-GFP mice displayed progressive behavioral impairment in open field and rotarod tests at 7 and 12 months of age [12]. Interestingly, in the colon of the mice, phosphorylated $\alpha$-syn was detected prior to the onset of motor behavioral deficits, indicating that the pathological alterations in the gut appear before the motor deficits even occur. The differential co-localization between $\alpha$-syn (-GFP) with calretinin, VIP and nNOS suggests that different subtypes of enteric neurons 
may possess a distinct role in gastrointestinal dysfunction in PD.

\section{Conclusions}

Using our BAC- $\alpha$-syn-GFP mouse model, we have demonstrated an age-dependent accumulation, phosphorylation and aggregation of $\alpha$-syn in the enteric neurons of the colon. Our model presents both the symptomatic and pathological features of early PD, expanding from the gut to the brain. The data indicate that this mouse model possesses the uniqueness and potential for studying PD gastrointestinal dysfunction.

\section{Additional files}

Additional file 1: Figure S1. A. The quenched a-syn-GFP signal, double immunofluorescence images labeled for PGP 9.5 and a-syn, and the phospho-a-syn immunohistochemical staining in the colon. (TIF 63429 kb)

Additional file 2: Figure S2. The gut transit time in the BAC and wild type male mice with age. (TIF26 kb)

\section{Abbreviations}

6-OHDA: 6-hydroxydopamine; BAC: Bacterial artificial chromosome; C57BL/ 6: c57 black 6; GFP: Green fluorescent protein; LB: Lewy bodies; LN: Lewy neurites; nNOS: neuronal nitric oxide synthase; PBS: Phosphate buffered saline; PD: Parkinson's disease; PFA: Paraformaldehyde; VIP: Vasoactive intestinal peptide; a-syn: a-synuclein

\section{Acknowledgements}

We would like to thank members of the Li lab for their intellectual inputs. We thank Mr. Sheng Yang and Mrs. Alicja Flasch for their technical support. We would also like to thank Dr. Andrew C. McCourt for the language editing

\section{Funding}

This work was supported by the National Natural Science Foundation (81430025) and also to the supports of the Swedish Research Council (K201561X-22297-03-4), EU-JPND (aSynProtec) and EU-JPND (REfreAME), EU H2020MSCA-ITN-2016 (Syndegen), BAGADILICO-Excellence in Parkinson and Huntington Research, the Strong Research Environment MultiPark (Multidisciplinary research on Parkinson's disease), the Swedish Parkinson Foundation (Parkinsonfonden), Torsten Söderbergs Foundation, Olle Engkvist Byggmästere Foundation. W.L. is supported by a scholarship from the China Scholarship Council.

\section{Availability of data and materials}

The materials used and/or analyzed during the current study are available from the corresponding author on reasonable request.

\section{Authors' contributions}

QQC and JYL conceived the study. QQC, CH and WL performed the experiments and data analyses. MTL, and ZYW provided intellectual inputs. $J Y L$ and QQC wrote the manuscript. All authors edited and approved the manuscript.

\section{Ethics approval and consent to participate}

All work involving animals was approved by the Ethical Committees for use of laboratory animals at Lund University, Sweden, and at Northeastern University, China.

\section{Competing interests}

The authors declare that they have no competing interests.

\section{Author details}

${ }^{1}$ Institute of Neuroscience, College of Life and Health Sciences, Northeastern University, 110819 Shenyang, People's Republic of China. ${ }^{2}$ Neural Plasticity and Repair Unit, Wallenberg Neuroscience Center, Department of Experimental Medical Science, Lund University, BMC A10, 22184 Lund, Sweden. ${ }^{3}$ Guangdong Province Key Laboratory of Brain Function and Disease, Zhongshan School of Medicine, Sun Yat-sen University, No. 74 Zhongshan Rd.2, Guangzhou 510080, China. ${ }^{4}$ Institute of Heath Sciences, China Medical University, 110112 Shenyang, People's Republic of China.

Received: 2 March 2018 Accepted: 6 June 2018

Published online: 30 June 2018

\section{References}

1. Hassler R. Zur Pathologie der Paralysis agitans und des postenzephalitischen Parkinsonismus. J Psychol Neurol. 1938;48:387-455

2. Lewy FH. Paralysis agitans: pathologische. In: Handbuch der Neurologie. Berlin: Julius Springer; 1912.

3. Tretiakoff C. Contribution a l'etude de l'anatomie pathologique du locus niger de Soemmering avec quelques deductions relatives a la pathogenie des troubles du tonus musculaire et de la maladie de Parkinson 1919.

4. Spillantini MG, Schmidt ML, Lee VM-Y, Trojanowski JQ, Jakes R, Goedert M. aSynuclein in Lewy bodies. Nature. 1997;388:839-40. https:/doi.org/10.1038/42166.

5. Schapira AHV, Tolosa E. Molecular and clinical prodrome of Parkinson disease: implications for treatment. Nat Rev Neurol. 2010;6:309-17. https:// doi.org/10.1038/nrneurol.2010.52

6. Holmqvist $\mathrm{S}$, Chutna O, Bousset L, Aldrin-Kirk P, Li W, Björklund T, et al. Direct evidence of Parkinson pathology spread from the gastrointestinal tract to the brain in rats. Acta Neuropathol. 2014;128:805-20. https://doi.org/ 10.1007/s00401-014-1343-6.

7. Braak H, de VRAI, Bohl J, Del Tredici K. Gastric alpha-synuclein immunoreactive inclusions in Meissner's and Auerbach's plexuses in cases staged for Parkinson's disease-related brain pathology. Neurosci Lett. 2006 396:67-72. https://doi.org/10.1016/j.neulet.2005.11.012

8. Braak H, Tredici KD, Rüb U, de VRAI, Jansen Steur ENH, Braak E. Staging of brain pathology related to sporadic Parkinson's disease. Neurobiol Aging. 2003;24:197-211. https://doi.org/10.1016/S0197-4580(02)00065-9

9. Svensson E, Horváth-Puhó E, Thomsen RW, Djurhuus JC, Pedersen L, Borghammer P, Sørensen HT. Vagotomy and subsequent risk of Parkinson's disease. Ann Neurol. 2015;78:522-9. https://doi.org/10.1002/ana.24448.

10. Schmidt RF, Gerhard T, editors. Human Physiology: Springer Science \& Business Media; 2013.

11. Willey JM, Sherwood L, Woolverton CJ. Prescott's microbiology. New York: McGraw-Hill; 2014.

12. Hansen C, Björklund T, Petit GH, Lundblad M, Murmu RP, Brundin P, Li J-Y. A novel a-synuclein-GFP mouse model displays progressive motor impairment, olfactory dysfunction and accumulation of a-synuclein-GFP. Neurobiol Dis. 2013;56:145-55. https://doi.org/10.1016/j.nbd.2013.04.017

13. Zhong C-B, Chen Q-Q, Haikal C, Li W, Svanbergsson A, Diepenbroek M, Li J-Y Age-Dependent Alpha-Synuclein Accumulation and Phosphorylation in the Enteric Nervous System in a Transgenic Mouse Model of Parkinson's Disease. Neurosci Bull. 2017;33:483-92. https://doi.org/10.1007/s12264-017-0179-1 .

14. Tsuboi K, Kimber TA, Shults CW. Calretinin-containing axons and neurons are resistant to an intrastriatal 6-hydroxydopamine lesion. Brain Res. 2000; 866:55-64. https://doi.org/10.1016/S0006-8993(00)02219-8 .

15. Mura A, Feldon J, Mintz M. The expression of the calcium binding protein calretinin in the rat striatum: effects of dopamine depletion and L-DOPA treatment. Exp Neurol. 2000;164:322-32. https://doi.org/10.1006/exnr.2000.7441

16. Kim BG, Shin DH, Jeon GS, Seo JH, Kim YW, Jeon BS, Cho SS. Relative sparing of calretinin containing neurons in the substantia nigra of 6-OHDA treated rat Parkinsonian model. Brain Res. 2000;855:162-5. https://doi.org/10. 1016/S0006-8993(99)02374-4

17. Padovan-Neto FE, Cavalcanti-Kiwiatkoviski R, Carolino RO, Anselmo-Franci J, Del Bel E. Effects of prolonged neuronal nitric oxide synthase inhibition on the development and expression of L-DOPA-induced dyskinesia in 6-OHDAlesioned rats. Neuropharmacology. 2015;89:87-99. https://doi.org/10.1016/j. neuropharm.2014.08.019

18. Yelkenli IH, Ulupinar E, Korkmaz OT, Şener E, Kuş G, Filiz Z, Tunçel N. Modulation of Corpus Striatal Neurochemistry by Astrocytes and Vasoactive Intestinal Peptide (VIP) in Parkinsonian Rats. J Mol Neurosci. 2016;59:280-9. https://doi.org/10.1007/s12031-016-0757-0 .

19. Bialecka M, Kurzawski M, Klodowska-Duda G, Opala G, Juzwiak S, Kurzawski $\mathrm{G}$, et al. CARD15 variants in patients with sporadic Parkinson's disease. Neurosci Res. 2007;57:473-6. https://doi.org/10.1016/j.neures.2006.11.012 . 
20. Hopfner F, Künstner A, Müller SH, Künzel S, Zeuner KE, Margraf NG, et al. Gut microbiota in Parkinson disease in a northern German cohort. Brain Res. 2017;1667:41-5. https://doi.org/10.1016/j.brainres.2017.04.019 .

21. Keshavarzian A, Green SJ, Engen PA, Voigt RM, Naqib A, Forsyth CB, et al. Colonic bacterial composition in Parkinson's disease. Mov Disord. 2015;30: 1351-60. https://doi.org/10.1002/mds.26307.

22. Sampson TR, Debelius JW, Thron T, Janssen S, Shastri GG, Ithan ZE, et al. Gut Microbiota Regulate Motor Deficits and Neuroinflammation in a Model of Parkinson's Disease. Cell. 2016;167:1469-1480.e12. https://doi. org/10.1016/j.cell.2016.11.018

Ready to submit your research? Choose BMC and benefit from:

- fast, convenient online submission

- thorough peer review by experienced researchers in your field

- rapid publication on acceptance

- support for research data, including large and complex data types

- gold Open Access which fosters wider collaboration and increased citations

- maximum visibility for your research: over $100 \mathrm{M}$ website views per year 